\title{
Group B streptococci infection model shows decreased regulatory capacity of cord blood cells
}

\author{
Kriszta Molnar ${ }^{1}$, Hannah Riedel ${ }^{1}$, Julian Schwarz ${ }^{1}$, Stefanie Dietz ${ }^{1}$, Bärbel Spring ${ }^{1}$, Laura Haag ${ }^{1}$, Christian F. Poets ${ }^{1}$, Christian Gille ${ }^{2 \bowtie}$ and \\ Natascha Köstlin-Gille ${ }^{1}$
}

(c) The Author(s) 2022

BACKGROUND: Sepsis is one of the leading causes of morbidity and mortality in the neonatal period. Compared to adults, neonates are more susceptible to infections, especially to systemic infections with Group B Streptococcus (GBS). Furthermore, neonates show defects in terminating inflammation. The immunological causes for the increased susceptibility to infection and the prolonged inflammatory response are still incompletely understood.

METHODS: In the present study, we aimed to investigate the reaction of cord blood mononuclear cells (MNC) to stimulation with GBS in comparison to that of MNC from adult blood with focus on the proliferative response in an in vitro infection model with heat-inactivated GBS.

RESULTS: We demonstrate that after stimulation with GBS the proliferation of T cells from adult blood strongly decreased, while the proliferation of cord blood T cells remained unchanged. This effect could be traced back to a transformation of adult monocytes, but not cord blood monocytes, to a suppressive phenotype with increased expression of the co-inhibitory molecule programmed death ligand 1 (PD-L1).

CONCLUSIONS: These results point towards an increased inflammatory capacity of neonatal MNC after stimulation with GBS. Targeting the prolonged inflammatory response of neonatal immune cells may be a strategy to prevent complications of neonatal infections.

Pediatric Research (2022) 92:1407-1416; https://doi.org/10.1038/s41390-021-01880-1

\section{IMPACT:}

- Neonatal sepsis often leads to post-inflammatory complications.

- Causes for sustained inflammation in neonates are incompletely understood.

- We show that cord blood T cells exhibited increased proliferative capacity after stimulation with group B streptococci (GBS) in comparison to adult $\mathrm{T}$ cells.

- Adult monocytes but not cord blood monocytes acquired suppressive activity and expressed increased levels of PD-L1 after GBS stimulation.

- Increased proliferative capacity of neonatal T cells and decreased suppressive activity of neonatal monocytes during GBS infection may contribute to prolonged inflammation and development of post-inflammatory diseases in newborns.

\section{INTRODUCTION}

Sepsis is one of the leading causes of morbidity and mortality in the neonatal period. ${ }^{1,2}$ The incidence of neonatal sepsis is about $0.05 \%$ in term neonates ( $\geq 37$ weeks of gestation) but increases to up to $36 \%$ among very low birth weight infants. ${ }^{3}$ Although decreasing in incidence, neonatal sepsis still causes approximately 400,000 infant deaths globally. ${ }^{4}$

Compared to adults, neonates and especially preterm infants are much more susceptible to infections, which is reflected in an eight times higher sepsis risk. ${ }^{5}$ Furthermore, the termination of inflammation seems to be disturbed in neonates and preterm infants, ${ }^{6}$ a phenomenon named sustained inflammation. ${ }^{7}$ This prolonged inflammatory reaction after initial activation of neonatal immune cells may contribute to the development of post-inflammatory diseases such as bronchopulmonary dysplasia (BPD) and periventricular leukomalacia (PVL), thereby significantly influencing long-term outcome. ${ }^{8-10}$ Both, increased susceptibility to infection as well as prolonged inflammation, can be traced back to altered reactions of neonatal immune cells compared to those of adults. ${ }^{11}$ Group B Streptococcus (GBS) is one of the leading cause of neonatal sepsis and meningitis. Although intrapartum antibiotic prophylaxis has significantly decreased the incidence of invasive GBS infections, still about 1 in 1000 newborns suffers from GBS sepsis often associated with a poor outcome. ${ }^{12,13}$ Some differences between neonates and adults in the reaction of immune cells to GBS have been identified ${ }^{14,15}$; however, it still

\footnotetext{
${ }^{1}$ Department of Neonatology, Tübingen University Children's Hospital, Calwerstrasse 7, 72076 Tübingen, Germany. ${ }^{2}$ Department of Neonatology, Heidelberg University Children's
} Hospital, Im Neuenheimer Feld 430, 69120 Heidelberg, Germany. ${ }^{\circledR}$ email: christian.gille@med.uni-heidelberg.de 
1408

remains unclear why neonates and especially preterm infants are much more susceptible to invasive GBS infections than adults and why they frequently develop post-inflammatory diseases.

In the present study, we aimed to examine the response of cord blood mononuclear cells (CBMC) to stimulation with GBS in comparison to that of mononuclear cells (MNC) isolated from peripheral blood (PB; peripheral blood mononuclear cells (PBMC)). In an in vitro co-culture model, we show (1) that the proliferation of PBMC strongly decreased after stimulation with GBS while proliferation of CBMC remained unchanged and that (2) the decreased proliferation of PBMC after GBS stimulation was due to changes in monocyte and T cell function. Also, (3) monocytes from adult, but not from $C B$, acquired suppressive activity and a different phenotype after stimulation with GBS. (4) T cells from adult blood expressed increased levels of the activation marker CD25 but did not acquire increased suppressive activity after stimulation with GBS.

\section{RESULTS \\ Decreased proliferation of adult blood but not CB MNC after stimulation with GBS}

First, we asked whether stimulation with GBS would influence $\mathrm{T}$ cell proliferation in PBMC and/or CBMC. PBMC and CBMC were isolated from adult or CB and stimulated with GBS for $18 \mathrm{~h}$. Unstimulated PBMC and CBMC served as controls. Subsequently, T cell proliferation was stimulated by addition of OKT3. We found that in adult donors, pre-stimulation with GBS led to a strong reduction in proliferation of $\mathrm{CD}^{+}{ }^{+} \mathrm{T}$ cells (median 20.0 versus $64.5 \%$ ) as well as $\mathrm{CD}^{+}$T cells (median 22.0 versus $71.5 \%$, both $n=18, p<0.0001$ ) in comparison to cells not stimulated with GBS. In contrast, stimulation of CB cells with GBS did not alter $\mathrm{CD}^{+}$or $\mathrm{CD}^{+} \mathrm{T}$ cell proliferation (median 52.0 versus $59.5 \%$ for $\mathrm{CD}^{+}{ }^{-} \mathrm{T}$ cell and 58.0 versus $67.0 \%$ for $\mathrm{CD}^{+}-\mathrm{T}$ cell proliferation) (Fig. $1 \mathrm{a}-\mathrm{d}$ ). Stimulation with lipoteiconic acid (LTA) induced similar effects with an inhibition of $\mathrm{CD}^{+}$- and $\mathrm{CD}^{+}-\mathrm{T}$ cell proliferation in PBMC and a tendency towards an increased $T$ cell proliferation in CBMC (Supplementary Fig. 2A-D).

\section{Decreased proliferation of adult blood MNC after GBS} stimulation is due to changes in monocyte and $\mathrm{T}$ cell function As OKT3-stimulated T cell proliferation in PBMC is a result of interaction between $T$ cells and antigen-presenting cells, we asked whether the decreased T cell proliferation in PBMC was due to changes in monocyte or T cell function. Therefore, we enriched monocytes and $T$ cells from PBMC by magnetic activated cell sorting (MACS) and stimulated them with GBS overnight. We then added either freshly isolated T cells (to pre-stimulated monocytes) or freshly isolated monocytes (to pre-stimulated T cells) from a different donor and stimulated the co-cultures with OKT3. Here we found that both, exclusive GBS pre-stimulation of monocytes (median 19.5\% (with GBS) versus $36.0 \%$ (without GBS) for $\mathrm{CD}^{+}{ }_{-}$ and $20.5 \%$ (with GBS) versus $52.5 \%$ (without GBS) for $\mathrm{CD}^{+}-\mathrm{T}$ cell proliferation, Fig. 2a, b) and exclusive GBS pre-stimulation of T cells (median $31.5 \%$ (with GBS) versus $47.5 \%$ (without GBS) for $\mathrm{CD}^{+}$- and $50 . \%$ (with $\mathrm{GBS}$ ) versus $61.0 \%$ (without $\mathrm{GBS}$ ) for $\mathrm{CD}^{+}{ }^{-} \mathrm{T}$ cell proliferation, all $n=6, p<0.05$, Fig. 2c, d), led to a decreased $\mathrm{CD}^{+}{ }^{-}$and $\mathrm{CD} 8^{+}-\mathrm{T}$ cell proliferation; however, the effect after GBS pre-stimulation of monocytes was stronger. As observed for stimulation of whole CBMC, exclusive stimulation of monocytes or $T$ cells from $C B$ did not significantly alter $T$ cell proliferation (Supplementary Fig. 3A-D).

\section{Monocytes from adult blood acquire suppressive activity after stimulation with GBS}

To further investigate functional changes in monocytes after GBS stimulation, we performed inhibition assays with GBS-stimulated monocytes and freshly isolated PBMC from a different donor with the hypothesis that GBS stimulation not only decreases the costimulatory capacity of adult monocytes but also induces suppressive capacity. Indeed, addition of GBS-stimulated monocytes from adult blood significantly inhibited $\mathrm{CD}^{+}$proliferation from median $75 \%$ (unstimulated adult monocytes) to median $37 \%$ (GBS-stimulated adult monocytes), while CB monocytes had no suppressive effect on $\mathrm{CD}^{+}-\mathrm{T}$ cell proliferation $(n=5, p<0.05$, Fig. $3 a, b) . C D 8^{+}-T$ cell proliferation was neither inhibited by adult nor by CB monocytes (Fig. 3c, d). Unstimulated monocytes from PB and CB had also no T cell-suppressive capacity (Supplementary Fig. 4).

\section{Monocytes from adult blood express increased levels of inhibitory molecules after GBS stimulation}

To get hints on potential mechanisms for this suppressive capacity, we analysed the expression of co-inhibitory molecules and effector enzymes associated with $T$ cell suppression. We found that, after stimulation with GBS, adult monocytes expressed $>100$ times higher levels of the co-inhibitory molecule PD-L1 than CB monocytes (median mean fluorescent intensity (MFI) 291 versus $26, n=9, p<0.0001$, Fig. $4 \mathrm{a}$, d), while expression of PD-L2 (Fig. 4a, e) and co-stimulatory molecules CD80 and CD86 (Fig. $4 a-c)$ were similar in adult and CB monocytes. Expression of arginase 1 (Argl) and indoleamine-2,3-dioxygenase (IDO), as well as the production of reactive oxygen species (ROS) did not differ between adult and CB monocytes after stimulation with GBS (Fig. 4a, f, h, i). Interestingly, CB monocytes expressed significantly higher levels of inducible nitric oxide synthase (iNOS) than adult monocytes (median MFI 171 versus 45, Fig. 4a, g).

T cells from adult blood express increased levels of CD25 after GBS stimulation

Lastly, we aimed to analyse the phenotype of $T$ cells after stimulation with GBS to identify a defect in adult $T$ cell activation as a potential reason for their decreased proliferative capacity after GBS stimulation. Interestingly, we found a higher expression of the activation marker CD25 on adult T cells after stimulation with GBS compared to neonatal T cells (median 36.5\% in PBMC versus $8.5 \%$ in CBMC, $n=6, p<0.005$, Fig. $5 a$, b), while the expression of CD69 did not differ between adult and neonatal cells (Fig. $5 \mathrm{a}, \mathrm{c}$ ). The increased expression of CD25 on adult cells was due to an increased CD25 expression on $\mathrm{CD}^{+}$(median $41.5 \%$ in PBMC versus $24.0 \%$ in CBMC, $n=8, p<0.005$, Fig. $5 d$, e) but not on $\mathrm{CD}^{+} \mathrm{T}$ cells (Fig. $5 \mathrm{~d}, \mathrm{f}$ ). Supplementary Fig. 5 shows the percentages of $\mathrm{CD}_{2} 5^{+}$cells from $\mathrm{CD}^{+}$and $\mathrm{CD}^{+} / \mathrm{CD}^{+}$cells without stimulation of GBS. Since CD25 is not only expressed on activated T cells but also on regulatory $T$ cells (Tregs), we analysed the proportion of $\mathrm{CD}^{+} / \mathrm{CD} 25^{+} / \mathrm{FoxP}^{+}$Tregs in adult blood and CB after stimulation with GBS but found no differences (Fig. $5 \mathrm{~g}, \mathrm{~h}$ ). Interestingly, addition of $\mathrm{CD} 4^{+} \mathrm{T}$ cells both from adult blood and from $C B$ to $P B M C$ from a different donor inhibited $T$ cell proliferation. Pre-stimulation with GBS increased the inhibitory capacity of $\mathrm{CD}^{+}{ }^{+}$cells; however, no differences between $\mathrm{CD}^{+}$ $T$ cells from adult and CB were observed (Fig. 5i, j).

\section{DISCUSSION}

Despite advances in neonatal care, sepsis in the first 28 postnatal days remains a major cause of mortality, leading to over 400,000 deaths per year. Knowledge concerning the cause(s) of this increased susceptibility to bacterial agents and on the regulation of neonatal immune functions is incomplete. Term and preterm infants with sepsis often develop long-term morbidity and neurodevelopmental impairment, such as BPD and PVL. This has been attributed to a prolonged inflammatory response following an initial activation of neonatal immune cells, called sustained inflammation. One of the most common pathogens associated with neonatal sepsis is GBS, counting for $40-50 \%$ of cases. ${ }^{1,16}$ 
a
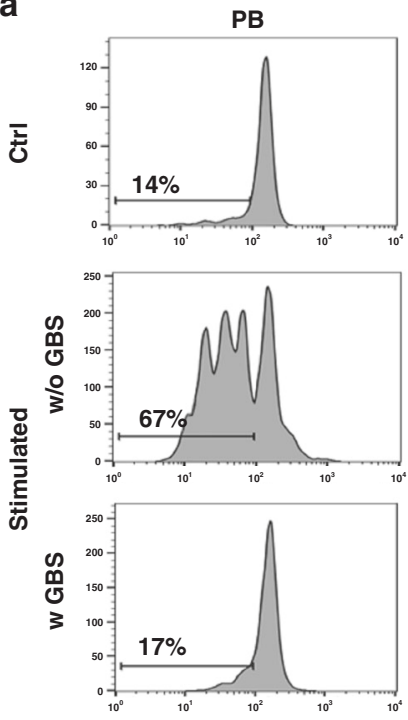

c
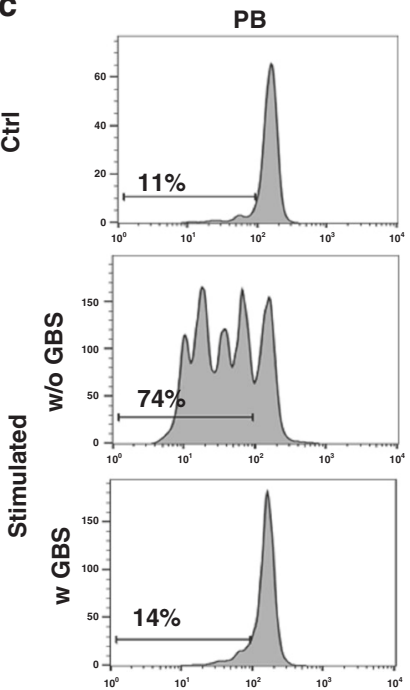

CB
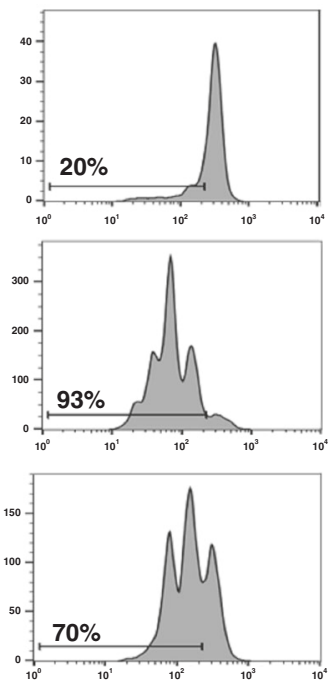

CB
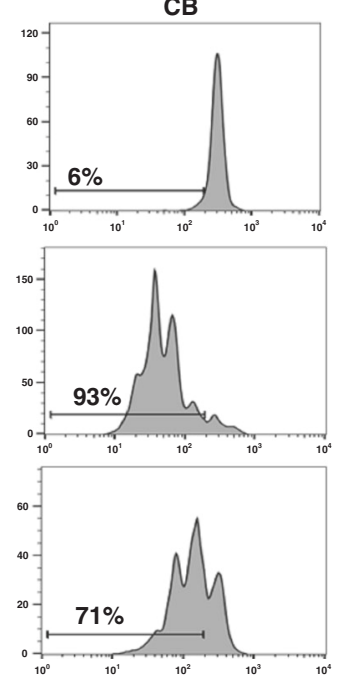

b

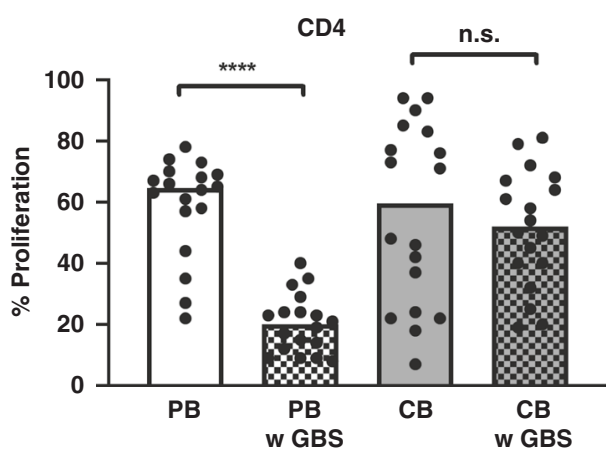

d

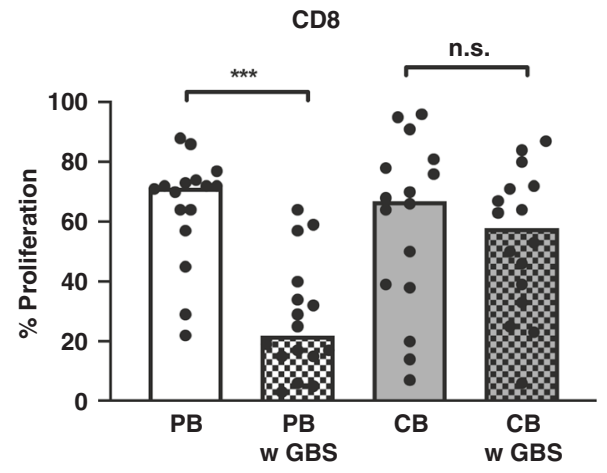

Fig. 1 Proliferation of adult and cord blood mononuclear cells after stimulation with GBS. CBMC and PBMC were isolated, stained with CFSE and stimulated with GBS overnight. The next day, cells were stimulated with OKT3. After another 3 days, T cell proliferation was assessed by CFSE dye dilution. T cells without pre-stimulation with GBS served as control. a, c Representative histogram plots show proliferation of CD4 ${ }^{+}-$T cells (a) and CD8 ${ }^{+}-\mathrm{T}$ cells (c) from adult (PB) and cord blood (CB) of unstimulated cells (ctrl), stimulated cells without (w/o GBS) and with additional stimulation with GBS (w GBS). b, d Scatter plots with bars show the percentage of proliferation of CD4 ${ }^{+}(\mathbf{b})$ and CD8 $8^{+}(\mathbf{d}) \mathrm{T}_{\text {cells }}$ in PBMC (white) and CBMC (grey) without (clean) or with (checked) stimulation with GBS. $n=18,{ }^{* * *} p<0.001,{ }^{* * * *} p<0.0001$, ns: not significant; Kruskal-Wallis test and Dunn's multiple comparison test.

First, we found that in adult cells, stimulation with GBS led to a strong reduction of $T$ cell proliferation, while proliferation of $C B$ $T$ cells remained unchanged. Several groups have shown that stimulation of immune cells from human adults or from adult mice with bacterial antigens or intact bacteria led to a suppressed $T$ cell proliferation. ${ }^{17-20}$ Only little data exist for neonates. Without bacterial stimulation, CB-MNC proliferate to a lesser extent than adult MNC, potentially due to a decreased co-stimulatory activity of $\mathrm{CB}$ monocytes. ${ }^{11}$ According to our present results, previous studies showed that proliferation of CBMC remained unaffected by stimulation with Escherichia coli but was decreased in adult PBMC. $^{6}$ Others described even a stimulatory effect of lipopolysaccharide on $\mathrm{T}$ cell proliferation in neonatal blood. ${ }^{21}$

In neonates, several innate immune functions like neutrophil chemotaxis, respiratory burst, extracellular trap formation ${ }^{22}$ and monocyte proinflammatory cytokine production ${ }^{23}$ seem to be decreased compared to adults. However, others, like phagocytosis, are adult-like even in very preterm infants. ${ }^{23}$ Similar observations have been made for neonatal adaptive immunity; while Th1responses to several pathogens and vaccines are decreased in neonates, they are able to develop adult-like Th1-responses to Bacillus Calmette-Guérin vaccination or cytomegalovirus infection. ${ }^{24}$ This indicates that the neonatal immune response is not generally affected and that the quality of the stimulus determines the response. Furthermore, it has been shown that there are different suppressive mechanisms present in $C B$, e.g. increased levels of immune-suppressive cells like myeloid-derived suppressor cells, ${ }^{25}$ immune-suppressive ${\mathrm{CD} 71^{+}}^{+}$erythroid cells ${ }^{26}$ and Tregs, ${ }^{27}$ as well as soluble factors like adenosine ${ }^{28}$ regulating the neonatal immune response. It may be speculated that the observed increased proliferative response of neonatal $T$ cells upon GBS stimulation reflects a high inflammatory capacity 
a

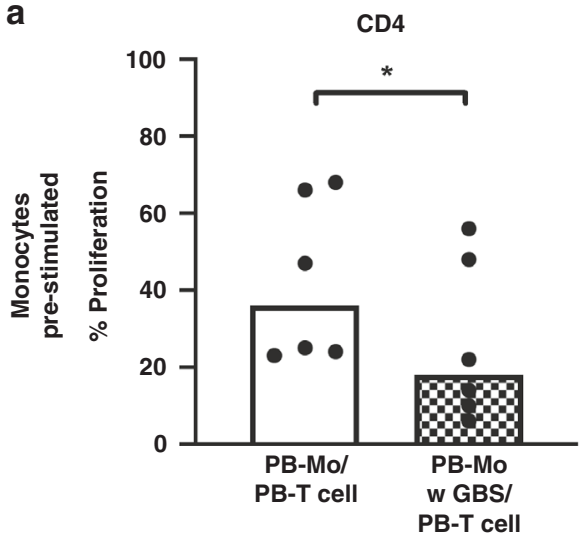

C

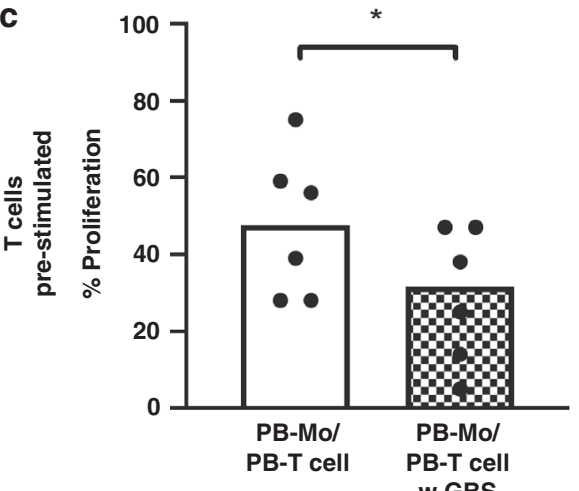

b
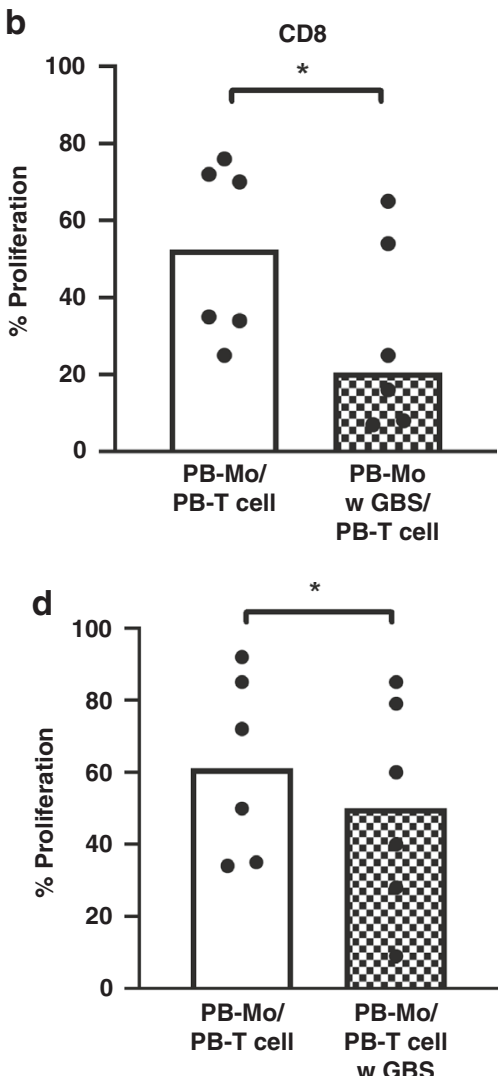

Fig. 2 T cell proliferation in adult blood after pre-stimulation of monocytes and T cells with GBS separately. Monocytes or T cells from PBMC were enriched by MACS and stimulated with GBS overnight. The next day, freshly isolated and CFSE-stained T cells from a different adult donor were added to pre-incubated monocytes $(\mathbf{a}, \mathbf{b})$ or pre-incubated, CFSE-stained T cells were added to freshly isolated monocytes from a different adult donor in a 2:1 ratio. Non-stimulated cells served as control. Co-cultures were stimulated with OKT3, and after $72 \mathrm{~h}$, T cell proliferation was assessed by flow cytometry. a, b Scatter diagrams with bars show proliferation of CD4 ${ }^{+}(\mathbf{a}, \mathbf{c})$ and CD8 ${ }^{+}(\mathbf{b}, \mathbf{d}) \mathrm{T}^{\mathrm{cell}}$ in $\mathrm{co}-$ culture with monocytes without (clean) or with (checked) pre-stimulation with GBS of monocytes (a, b) or T cells (c, d). $n=6,{ }^{*} p<0.05, \mathrm{~ns}$ : not significant; Wilcoxon matched-pairs signed rank test.

regulated by suppressive mechanisms under steady state conditions, but resulting in a decreased capacity to terminate inflammation during infection, potentially leading to PVL or BPD. We further found that the decreased proliferation of adult MNC after stimulation with GBS was due to changes in monocyte as well as $T$ cell functions. Adult monocytes acquired suppressive capacity upon stimulation with GBS while CB monocytes did not. The induction of suppressive monocytes during infection in adults has been repeatedly described. ${ }^{29,30}$ For neonatal cells, some studies described reduced expression of pro-inflammatory ${ }^{14,31}$ and increased expression of anti-inflammatory cytokines. ${ }^{32}$ This was traced back to high levels of soluble immune-modulatory factors like adenosine, prostaglandins and sex hormones 33,34 perinatally. Contrarily, Roger et al. showed that the proinflammatory macrophage migration inhibitory factor MIF contributed to detrimental hyperinflammation during sepsis. ${ }^{35}$ Similarly, Heinemann et al. found a specific population of inflammatory monocytes in neonates that are regulated/ suppressed by S100A8/A9 alarmins. ${ }^{36}$ Together with our present results, it can be assumed that neonatal monocytes do not have an intrinsically decreased inflammatory capacity but that this is rather controlled by extrinsic factors.

We could not fully uncover the mechanism through which adult monocytes acquire suppressive properties upon GBS stimulation. We found a strong upregulation of PD-L1 in adult but not in neonatal monocytes. This is in line with a previous study showing increased PD-L1 expression on monocytes from septic patients and restoration of monocyte functions during sepsis after blockade of PD-L1. ${ }^{37}$ In vitro studies revealed that stimulation of monocytes from healthy adults with streptococcal pyrogenic exotoxin A facilitated the accumulation of Tregs through PD-L1 ${ }^{38}$ pointing towards a regulatory role of PD-L1 expression on monocytes during infection in adults. ${ }^{39}$ In the present study we did not investigate any downstream pathways of PD-L1 activation, thus it can only be speculated that the increased PD-L1-expression in adult monocytes in comparison to $C B$ monocytes contributes to the acquisition of inhibitory capacity. Expression of PD-L1 on neonatal monocytes upon bacterial stimulation has not yet been investigated. However, it seems to be relevant for the development of tolerance to allergens in the first weeks of life. ${ }^{40}$ Further studies are needed to investigate the role of PD-L1 for neonatal sepsis and pathogenesis of post-inflammatory diseases.

Interestingly, we found no differences in the expression of Argl and IDO, as well as in the production of ROS between adult monocytes and CB monocytes after stimulation with GBS that could be responsible for their $T$ cell-suppressive activity. ${ }^{41}$ One previous study described a lower baseline expression of IDO in CB monocytes than adult monocytes. ${ }^{42}$ However the overall expression of IDO in monocytes in this study was very low $(<3 \%)$, while in our study all monocytes expressed IDO, indicating that IDO needs stimulation to be expressed and differences can only be detected after stimulation. Instead, we even found an increased expression of iNOS in CB monocytes in comparison to adult cells, indicating that under our conditions NO production is not primarily 
a
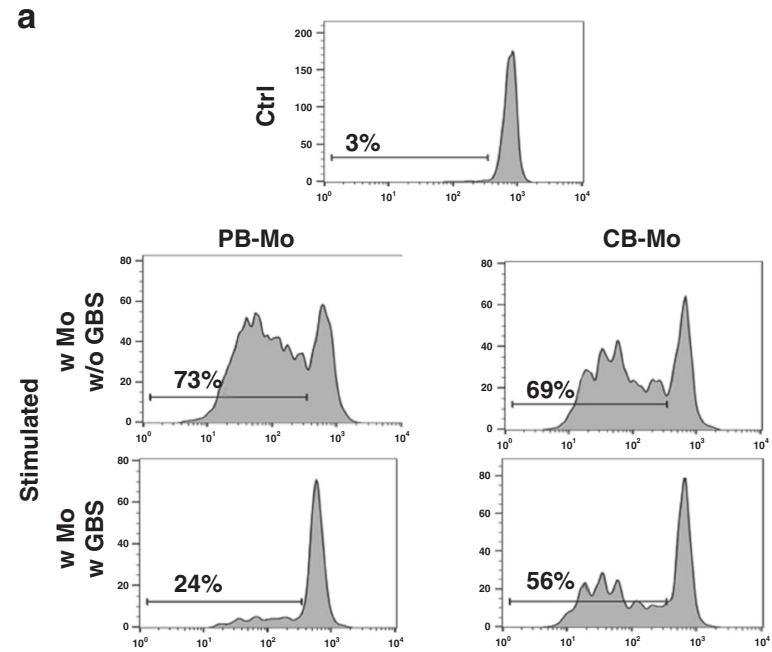

C
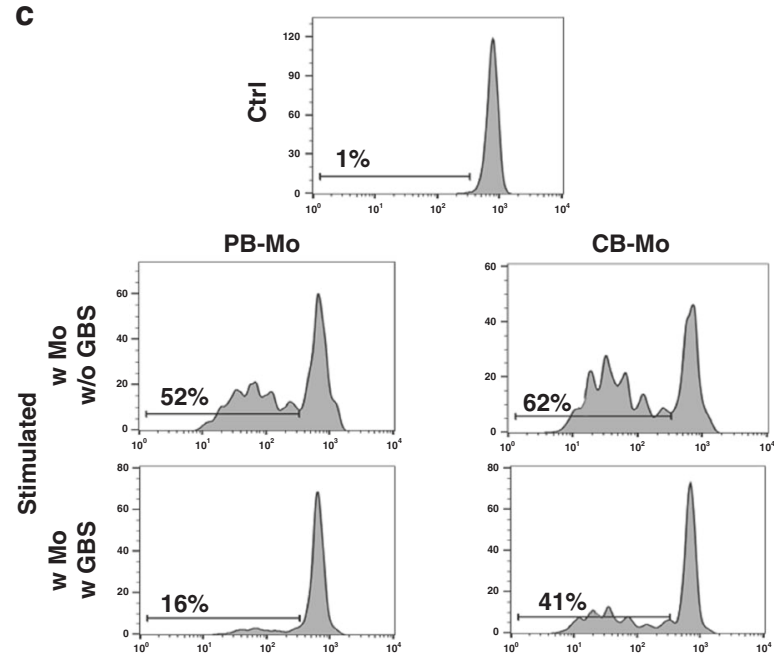

b

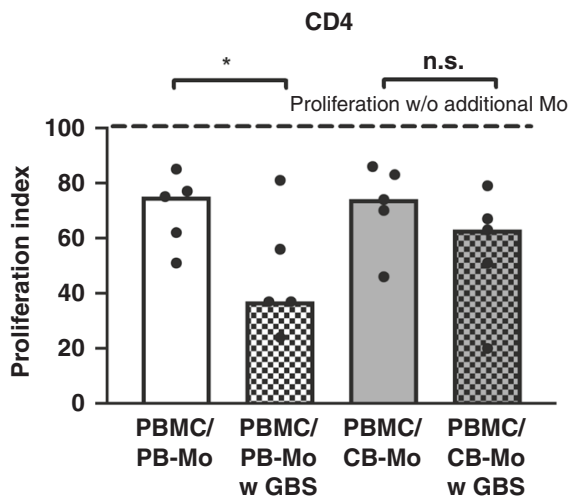

d

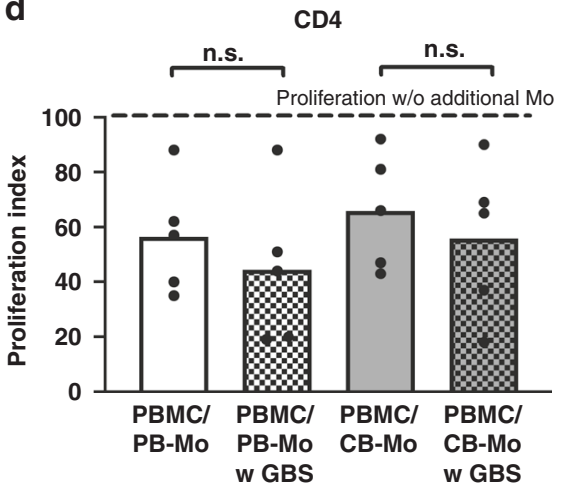

Fig. 3 Inhibition of T cell proliferation by GBS-stimulated monocytes. Monocytes were enriched from CBMC/PBMC by MACS and stimulated with GBS overnight. Monocytes without GBS stimulation served as control. The next day, monocytes were added to freshly isolated, CFSEstained and OKT3/IL-2-stimulated PBMC. After 4 days, T cell proliferation was assessed by CFSE dye dilution. Proliferation index was determined as the ratio of $T$ cell proliferation with and without addition of monocytes. $\mathbf{a}$, $\mathbf{c}$ Representative histogram plots show proliferation of $\mathrm{CD}^{+} \mathrm{T}$ cells $(\mathbf{a})$ and $\mathrm{CD}^{+} \mathrm{T}$ cells (c) after addition of monocytes from PB and from CB of unstimulated cells (ctrl) and stimulated cells without (w/o GBS) and with additional stimulation with GBS (w GBS). b, d Bar graphs show the inhibitory effect of monocytes on proliferation of $\mathrm{CD}^{+}(\mathbf{b})$ and $\mathrm{CD} 8^{+} \mathrm{T}$ cells (c). Dashed line shows proliferation of target PBMC without addition of monocytes. White/grey bars show T cell proliferation after addition of adult/cord blood monocytes. Clean bars show the effect of monocytes without and checked bars with prestimulation with GBS. $n=6,{ }^{*} p<0.05$, ns not significant; Friedman test and Dunn's multiple comparison test.

responsible for their suppressive activity. An increased NO production upon GBS infection/stimulation was shown to mediate disruption of blood-brain barrier and lung inflammation. ${ }^{43}$ After neonatal hypoxic-ischaemic brain injury, peripheral monocytes infiltrate the brain and contribute to brain injury. ${ }^{44}$ Monocytes also influence the onset of BPD. ${ }^{45}$ Thus, the increased iNOS expression in neonatal monocytes may contribute to the development of PVL and BPD and needs further investigations.

Lastly, we found that $\mathrm{T}$ cells and especially $\mathrm{CD} 4^{+} \mathrm{T}$ cells from adult blood expressed increased levels of the activation marker CD25 after stimulation with GBS in comparison to T cells from CB. CD25 is part of the receptor for interleukin-2 (IL-2R) and IL-2R knockout mice exhibit lymphoproliferation and severe autoimmunity. ${ }^{46}$ This is in line with our results of an increased proliferation of neonatal $\mathrm{T}$ cells lacking CD25 expression in comparison to adult cells. Furthermore, CD25 is expressed on Tregs, and IL-2 is required for Treg induction from conventional T cells. ${ }^{46}$ However, levels of Tregs in cultures after stimulation with GBS did not differ between PBMC and CBMC and there were no relevant differences in suppressive capacity between adult and $\mathrm{CB} \mathrm{CD4}{ }^{+} \mathrm{T}$ cells after stimulation with GBS. Interestingly, stimulation with GBS induced suppressive capacity in $\mathrm{CD}^{+}$ $T$ cells isolated from CBMC and PBMC. We assume that a functional activation of Tregs by GBS stimulation is responsible for this effect. However, this functional activation seems not specific to CBMC but occurs to the same extent in PBMC. Further investigations are needed to elucidate the impact of decreased upregulation of CD25 in neonatal T cells and its contribution to immune dysfunction during neonatal sepsis.

A limitation of our study is that nothing was known about the GBS status of mothers of the infants included in this study. Thus, it cannot be excluded that a certain pre-activation of CB cells had taken place which could explain the variability in results obtained from CB. Furthermore, CB collection was anonymized and no information on sex, exact gestational age or birth mode was available-all factors that may also influence the results. 


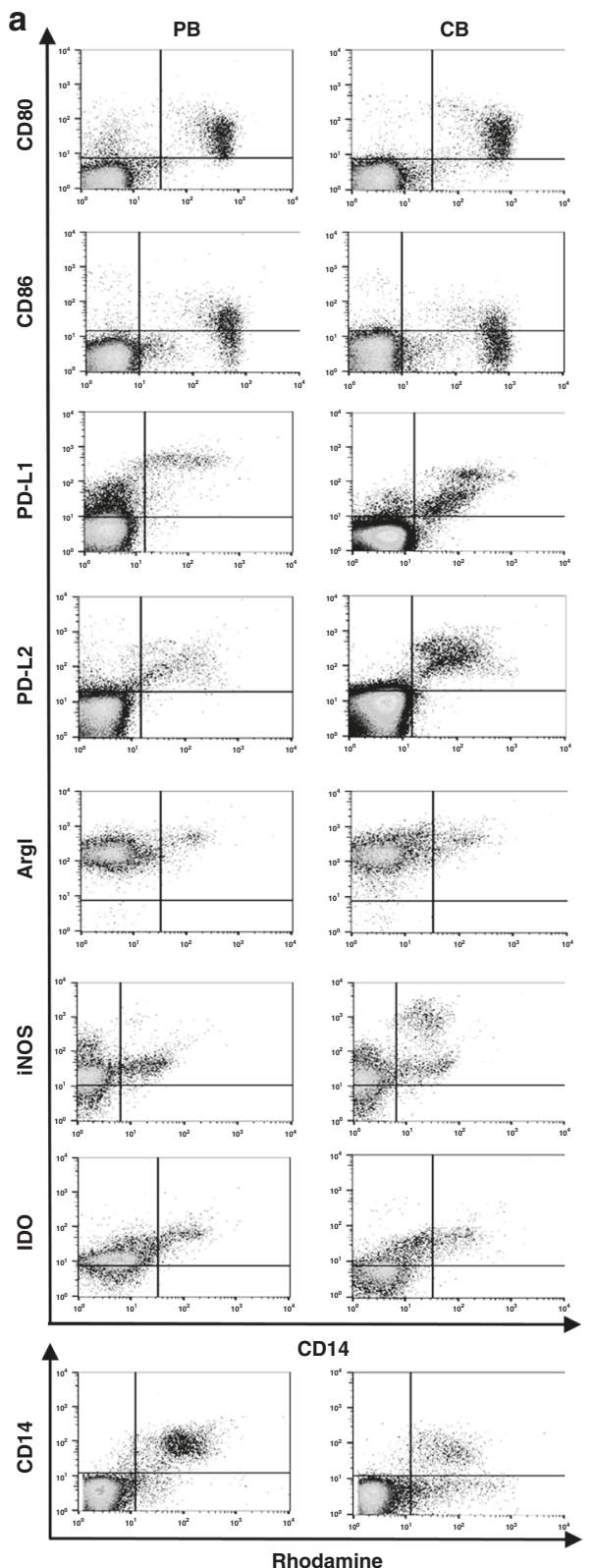

b
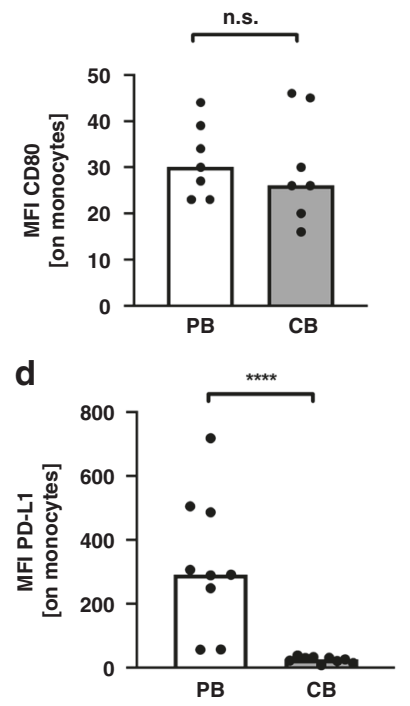

f

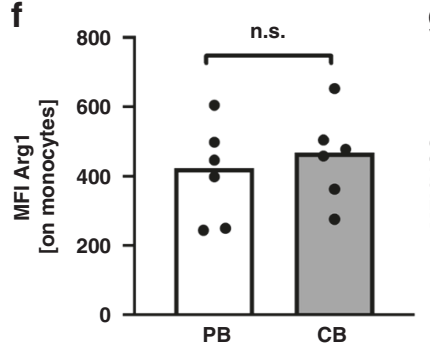

h

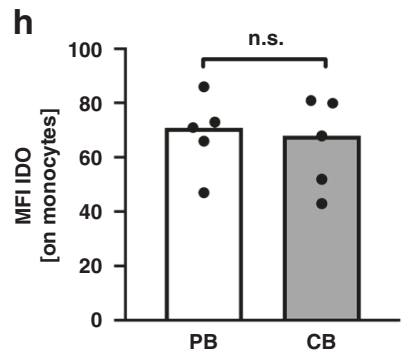

C
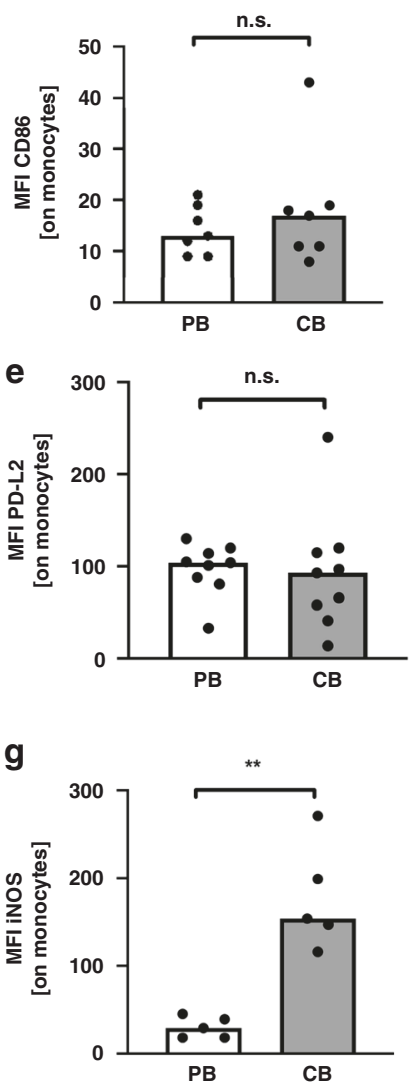

i

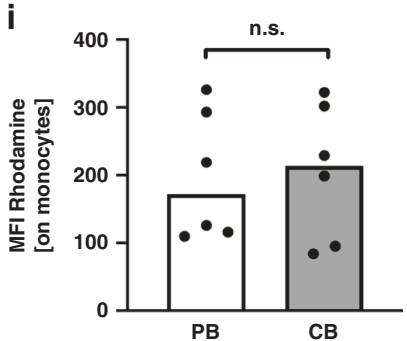

Fig. 4 Expression of surface molecules and effector enzymes on monocytes after stimulation with GBS. CBMC and PBMC were isolated and incubated with GBS overnight. Expression of surface molecules, intracellular effector enzymes, and reactive oxygen production was determined by flow cytometry. a Representative density plots show the expression of CD80, CD86, PD-L1, PD-L2, Argl, iNOS, IDO and Rhodamine ( $y$-axis) on $\mathrm{CD} 14^{+}$adult (PB) and cord blood (CB) monocytes ( $x$-axis). b-i Scatter plots with bars show the mean fluorescent intensity (MFI) for the expression of CD80 (b), CD86 (c), PD-L1 (d), PD-L2 (e) Argl (f), iNOS (g), IDO (h) on and the production of ROS (i) by adult (white) and cord blood (grey) monocytes after stimulation of GBS. $n=5-9$, ${ }^{* *} p<0.01,{ }^{* * *} p<0.0001$, ns not significant; Mann-Whitney test.

Another limitation is that an immune maturation takes place over the first weeks of life that is influenced by various factors, for example breastfeeding. ${ }^{47}$ Since we used CB for our analyses, the influences that play a role after birth could not be considered.

In conclusion, we show that stimulation with GBS had a strong effect on $\mathrm{CD}^{+}$and $\mathrm{CD}^{+}{ }^{+}$cell proliferation in adult, but not in $\mathrm{CB}$ cells, accompanied by the acquisition of suppressive capacity and upregulation of PD-L1 in adult monocytes. Our study illustrates a novel in vitro effect by which GBS may influence neonatal immune cells. Further studies are needed to uncover the underlying mechanisms and to evaluate if these effects also play a role in vivo. Altered reactions of neonatal immune cells to GBS may not only influence the pathogenesis of neonatal sepsis, but may also contribute to the development of post-inflammatory diseases like BPD and PVL. Modulating the post-inflammatory immune regulation may be an opportunity to prevent complications of inflammation in neonates.

\section{METHODS}

\section{Patients}

The Ethics Committee of Medical Faculty of the University of Tübingen approved this study (458/2019BO1), and CB was collected from healthy term newborns ( $\geq 37+0$ gestational weeks, total $n=54$ ) immediately after Caesarean section or vaginal delivery in a syringe and anticoagulated with 

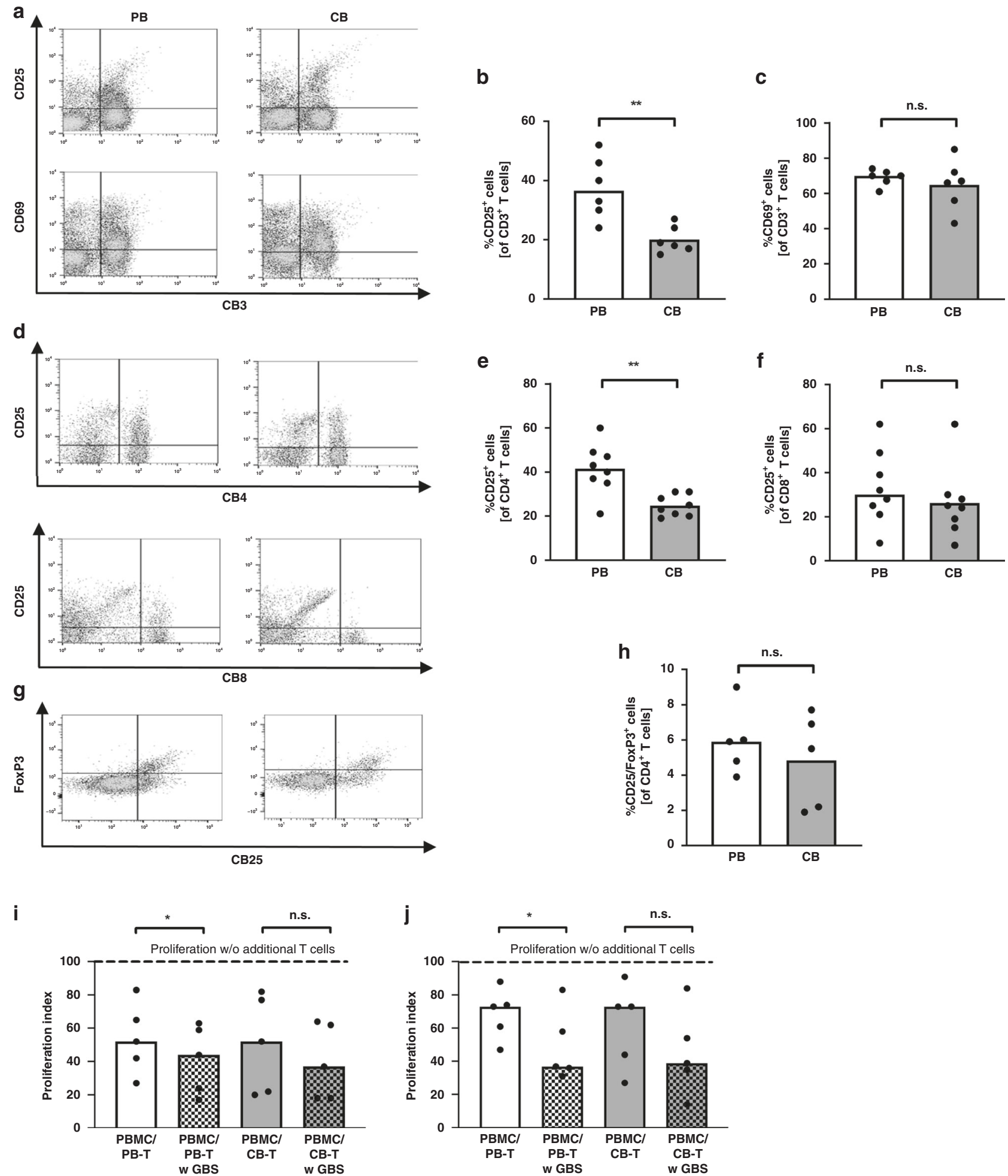

heparin. Collection of CB samples was anonymized, thus, no information on sex, exact gestational age or birth mode was available. Parents gave their written informed consent. Children with intra-amniotic infection (defined according to the guideline of the German Society for Gynaecology and Obstetrics (DGGG) as maternal fever $\left(\geq 38.0^{\circ} \mathrm{C}\right)$, increased maternal inflammatory markers without any other cause (C-reactive protein $>10 \mathrm{mg} / \mathrm{l}$ or elevation of white blood cell count $>15,000 / \mu \mathrm{l})$, foetal or maternal tachycardia, painful uterus and foul-smelling amniotic fluid) were excluded. PB from healthy adults was collected from adult volunteers (total $n=54$ ).

\section{Cell isolation and culture}

MNC were isolated by density gradient centrifugation according to previously described protocols. ${ }^{48}$ Heparinized whole blood was diluted in phosphatebuffered saline (PBS) to a total volume of $35 \mathrm{ml}$ and added carefully onto 15 $\mathrm{ml}$ lymphocyte separation solution (Biochrom $\mathrm{GmbH}$, Berlin, Germany). Cells were centrifuged and the MNC layer was collected. Cell count was determined and cells were diluted in PBS at a concentration of $2 \times 10^{6}$ cells $/ \mathrm{ml}$ for extracellular staining or at appropriate concentrations (see below) in RPMI supplemented with $10 \%$ foetal calf serum (FCS), $1 \%$ penicillin/ streptomycin and $1 \%$ Glutamine for culturing and functional analyses. 
Fig. 5 Expression of surface molecules on T cells after stimulation with GBS and inhibition of T cell proliferation by GBS-treated T cells. CBMC and PBMC were isolated and incubated with GBS overnight. Cells without GBS stimulation served as control. Expression of surface molecules (a-f) or intracellular FoxP3 $(\mathbf{g}, \mathbf{h})$ on T cells was determined by flow cytometry. Pre-incubated T cells were added to freshly isolated, CFSE-stained and OKT3/IL-2-stimulated PBMC. After 4 days, T cell proliferation was assessed by CFSE dye dilution. Proliferation index was determined as the ratio of $T$ cell proliferation with and without addition of $T$ cells $(\mathbf{i}, \mathbf{j})$. Representative density plots show the expression of CD25 and CD69 on CD3 ${ }^{+}$T cells (a), the expression of CD25 on $\mathrm{CD}^{+} / \mathrm{CD} 4^{+}$and $\mathrm{CD} 3^{+} / \mathrm{CD}^{+} \mathrm{T}$ cells (d), and the expression of CD25 and FoxP3 on $\mathrm{CD}^{+} / \mathrm{CD}^{+} \mathrm{T}$ cells $(\mathbf{g})$ from adult peripheral blood (PB) and cord blood (CB). Cells in d were pre-gated on CD3 and cells in panel $\mathbf{g}$ were pre-gated on CD3 and CD4. Scatter plots with bars show the percentage of CD3 T-cells expressing CD25 (b) or CD69 (c) percentage of CD25 on $\mathrm{CD}^{+} / \mathrm{CD}^{+}(\mathbf{e}), \mathrm{CD}^{+} / \mathrm{CD}^{+}$(f) and percentage of CD25 and FoxP3 expresssion on CD3 and CD4 T-cells, adult (white) and cord blood (grey) T cells after stimulation of GBS. Scatter blot $n=6-8,{ }^{* *} p<0.01$, ns not significant; Mann-Whitney test. Bar graphs show the inhibitory effect on proliferation of $\mathrm{CD}^{+}$(i) and CD8 ${ }^{+} \mathrm{T}$ cells $(\mathbf{j})$. Dashed line shows the proliferation of target PBMC without addition of T cells. White/grey bars show $\mathrm{T}$ cell proliferation after addition of adult/cord blood T cells. Clean bars show the effect of T cells without and checked bars with prestimulation with GBS. $n=5,{ }^{*} p<0.05$, ns not significant; Friedman test and Dunn's multiple comparison test.

For separation of $\mathrm{T}$ cells and monocytes from the PBMC and CBMC fraction, cells were labelled with human $\mathrm{CD}^{+} \mathrm{T}$ cell isolation kit or human Pan Monocyte Isolation kit (Miltenyi Biotec, Bergisch Gladbach, Germany) and separated by MACS in an autoMACS ${ }^{\circledR}$ separator according to the manufacturer's protocol (Miltenyi Biotec).

For stimulation with GBS or LTA, cells were seeded in $500 \mu \mathrm{l}$ of RPMI supplemented with $10 \%$ FCS, $1 \%$ penicillin/streptomycin and $1 \%$ Glutamine at a concentration of $1 \times 10^{6}$ cells $/ \mathrm{ml}$ in 48-well plates (Greiner Bio-one, Kremsmünster, Austria). Cells were stimulated either with GBS in a multiplicity of infection (MOI) of 1:50 or with LTA $(1 \mu \mathrm{g} / \mathrm{ml}$, Sigma, Munich, Germany) for $18 \mathrm{~h}$. Unstimulated cells served as control.

\section{Bacterial culture}

The culture of Group B streptococci was performed as previously described. ${ }^{49}$ Briefly, the GBS strain BSU98 was freshly grown on Columbia agar plates (Sigma, Munich, Germany) supplemented with $5 \%$ defibrinated sheep blood and spectinomycin ( $150 \mathrm{~g} / \mathrm{ml}$; Sigma) for $16 \mathrm{~h}$. Colonies were re-suspended in PBS and bacterial number was determined spectrometrically. Bacteria were inactivated for $30 \mathrm{~min}$ at $70^{\circ} \mathrm{C}$. For stimulation of PBMC and CBMC, inactivated GBS were used in a MOI of 1:50.

\section{Flow cytometry}

Antibodies used for extracellular staining of monocytes and T cells were purchased from BD Bioscience, Heidelberg, Germany (CD3-PerCp clone SK7, CD4-APC clone RPA-T4, CD8-PE clone SK1, CD14-APC clone M $4 P 9$, CD25-FITC clone 2A3, CD80-PE clone L307.4, CD86-PerCp clone 2331), Miltenyi Biotec (CD69-APC clone REA-824) and BioLegend, San Diego, CA (PD-L1-APC clone B7H-1, PD-L2-PE clone MIH1).

For intracellular staining, a total of $2 \times 10^{5}$ extracellularly stained PBMC and CBMC were washed with FACSbuffer (PBS with $0.1 \%$ FCS, Sigma) and $0.1 \%$ sodium azide (Sigma). One hundred microlitres of Cytofix/Cytoperm (BD Pharmingen, Heidelberg, Germany) were added and cells were incubated for $10 \mathrm{~min}$ at $37^{\circ} \mathrm{C}$. After that, intracellular antibodies for Argl (Argl-PE clone 658922), IDO (IDO-PE clone 700838) (both R\&D Systems, Wiesbaden-Nordenstadt, Germany) and iNOS (iNOS-Alexa Fluor 647 clone C-11; Santa Cruz, Heidelberg, Germany) were added and incubated for another $10 \mathrm{~min}$ at $37^{\circ} \mathrm{C}$. Cells were then washed twice with fluorescenceactivated cell sorting (FACS) buffer and analysed by flow cytometry. Data acquisition was performed with a FACS Calibur flow cytometer and data were analysed via CellQuest (BD Biosciences). ${ }^{48}$

All antibodies were tested for their specificity by isotype control staining, when introduced in our laboratory. Isotype control antibodies used for intracellular staining of effector enzymes were Alexa Fluor 647 mouse lgG1 IC (for iNOS), PE mouse lgG2b IC (for Argl) and PE mouse IgG1 IC (for IDO) (all from BD Bioscience). For each antibody combination, compensation with single staining was performed. For analysis of expression of costimulatory and co-inhibitory molecules on and expression of effector enzymes by monocytes as well as activation markers on T cells, FMO controls were run for each sample.

\section{ROS detection}

For detection of ROS, $1 \times 10^{6}$ PBMCs and CBMCs, pre-incubated without or with GBS in a MOI of 1:50 for $18 \mathrm{~h}$, were incubated with dihydrorhodamine 123 (Sigma) in PBS for $5 \mathrm{~min}$ in a water bath at $37^{\circ} \mathrm{C}$. After that, cells were stimulated for $10 \mathrm{~min}$ with $60 \mathrm{ng} / \mathrm{ml}$ of phorbol 12-myristate 13-acetate
(Sigma). Cells were washed, surface staining with anti-CD14-APC (BD Biosciences) was performed and ROS production was analysed by flow cytometry.

\section{T cell proliferation assay}

For analysis of T cell proliferation after GBS stimulation, freshly isolated CBMC and PBMC were stained with carboxyfluorescein-succinimidyl ester (CFSE, Invitrogen, Heidelberg, Germany) according to the manufacturer's instructions, seeded into round-bottom CD96-well plates $\left(2 \times 10^{5}\right.$ cells in $100 \mu \mathrm{l}$ media) and stimulated with GBS in a MOI of 1:50 for $18 \mathrm{~h}$. Afterwards, $0.01 \mu \mathrm{g} / \mathrm{ml} \mathrm{OKT3} \mathrm{(Invitrogen/Thermo} \mathrm{Fisher,} \mathrm{Waltham,} \mathrm{MA)} \mathrm{was}$ added. After $72 \mathrm{~h}$ of incubation, cells were harvested and stained with antiCD4-APC and anti-CD8-PE. CFSE fluorescence intensity was analysed by flow cytometry. Apoptosis rates after 4 days of culture were assessed by Annexin V staining (Annexin-V-APC, BD Biosciences) and flow cytometry and revealed apoptosis rates of about 5-10\% (Supplementary Fig. 1).

To analyse the impact of GBS stimulation on monocytes and T cells separately, isolated monocytes or T cells from PB and CB (each $1 \times 10^{5}$ cells in $500 \mu \mathrm{l}$ media in 48 well plates) were cultured alone or with GBS for $18 \mathrm{~h}$ (MOI 1:50). Cells were removed from the plates and cell count was assessed in a Neubauer counting chamber. In order to maintain allogeneic combinations both in $\mathrm{CB}$ and in adult blood, we always used different donors for monocytes and T cells. The next day, the counterpart of cells was isolated freshly from another donor.

Pre-cultured monocytes were added in a 1:2 ratio to $2 \times 10^{5}$ freshly isolated $\mathrm{T}$ cells from a different adult donor stained with CFSE and stimulated with $0.01 \mu \mathrm{g} / \mathrm{ml}$ OKT3 in a round-bottom 96-well plate. As control, only RPMI 1640 with supplements was added to the CFSE-stained cells. After $72 \mathrm{~h}$, cells were harvested and stained with anti-CD4-APC and anti-CD8-PE.

Pre-cultured T cells were labelled with CFSE and stimulated with $0.01 \mu \mathrm{g} /$ $\mathrm{ml} \mathrm{OKT3.} \mathrm{Freshly} \mathrm{isolated} \mathrm{monocytes} \mathrm{from} \mathrm{a} \mathrm{different} \mathrm{adult} \mathrm{donor} \mathrm{were}$ added as co-stimulatory cells in a 1:2 ratio to $2 \times 10^{5} \mathrm{~T}$ cells in a roundbottom 96-well plate. After $72 \mathrm{~h}$ of incubation, cells were harvested and stained with anti-CD4-APC and anti-CD8-PE. CFSE fluorescence intensity was analysed by flow cytometry. Data acquisition was performed with a FACSCalibur flow cytometer and data were analysed via CellQuest (BD Biosciences).

To analyse the suppressive capacity of GBS stimulated monocytes or $T$ cells, isolated monocytes or T cells from PB and CB (each $1 \times 10^{5}$ cells in $500 \mu \mathrm{l}$ media in 48-well plates) were cultured alone or with GBS for $18 \mathrm{~h}$ (MOI 1:50). Cells were removed from the plates and cell count was assessed in a Neubauer counting chamber. The next day, PBMC were isolated freshly from another donor. Pre-cultured monocytes or precultured T cells were added in a 1:2 ratio to $2 \times 10^{5}$ freshly isolated PBMC from a different adult donor stained with CFSE and stimulated with 0.01 $\mu \mathrm{g} / \mathrm{ml}$ OKT3 and $100 \mathrm{U} / \mathrm{ml} \mathrm{IL-2} \mathrm{(R \& D} \mathrm{Systems)} \mathrm{in} \mathrm{a} \mathrm{round-bottom} \mathrm{96-well}$ plate. As control, only RPMI1640 with supplements was added to the CFSEstained cells. After $72 \mathrm{~h}$, cells were harvested and stained with anti-CD4APC and anti-CD8-PE.

\section{Statistics}

Statistical analysis was done with GraphPad Prism version 8.0. Values were tested for Gaussian distribution using D'Agostino and Pearson omnibus normality test. Differences in proliferation assays and inhibition assays with comparison of more than two groups were analysed by Friedman test and Dunn's multiple comparison test. Differences in proliferation assays with 
comparison of two groups were analysed using the Wilcoxon matchedpaired signed rank test. Differences in the expression of surface molecules and intracellular molecules were determined using Mann-Whitney test. A $p$ value $<0.05$ was considered significant.

\section{REFERENCES}

1. Shane, A. L., Sanchez, P. J. \& Stoll, B. J. Neonatal sepsis. Lancet 390, 1770-1780 (2017).

2. Achten, N. B. et al. Association of use of the neonatal early-onset sepsis calculator with reduction in antibiotic therapy and safety: a systematic review and metaanalysis. JAMA Pediatr. 173, 1032-1040 (2019).

3. Dong, Y. \& Speer, C. P. Late-onset neonatal sepsis: recent developments. Arch. Dis. Child. Fetal Neonatal Ed. 100, F257-F263 (2015).

4. Puopolo, K. M., Benitz, W. E., Zaoutis, T. E., Committee On Fetus and Newborn \& Committee On Infectious Diseases. Management of neonates born at $\leq 34$ 6/ 7 weeks' gestation with suspected or proven early-onset bacterial sepsis. Pediatrics 142, e20182896 (2018).

5. Stichtenoth, G. et al. Major contributors to hospital mortality in very-low-birthweight infants: data of the birth year 2010 cohort of the German Neonatal Network. Klin. Padiatr. 224, 276-281 (2012).

6. Gille, C. et al. The CD95/CD95L pathway is involved in phagocytosis-induced cell death of monocytes and may account for sustained inflammation in neonates. Pediatr. Res. 73, 402-408 (2013).

7. Dammann, O. \& Leviton, A. Intermittent or sustained systemic inflammation and the preterm brain. Pediatr. Res. 75, 376-380 (2014).

8. Levy, O. Impaired innate immunity at birth: deficiency of bactericidal/permeability-increasing protein (BPI) in the neutrophils of newborns. Pediatr. Res. 51, 667-669 (2002).

9. Jones, C. A. et al. Undetectable interleukin (IL)-10 and persistent IL-8 expression early in hyaline membrane disease: a possible developmental basis for the predisposition to chronic lung inflammation in preterm newborns. Pediatr. Res. 39, 966-975 (1996).

10. Blahnik, M. J., Ramanathan, R., Riley, C. R. \& Minoo, P. Lipopolysaccharide-induced tumor necrosis factor-alpha and IL-10 production by lung macrophages from preterm and term neonates. Pediatr. Res. 50, 726-731 (2001).

11. Orlikowsky, T. W. et al. Expression and regulation of B7 family molecules on macrophages (MPhi) in preterm and term neonatal cord blood and peripheral blood of adults. Cytom. B Clin. Cytom. 53, 40-47 (2003).

12. Berardi, A. et al. Group B streptococcus late-onset disease: 2003-2010. Pediatrics 131, e361-e368 (2013)

13. Fluegge, $K$. et al. Incidence and clinical presentation of invasive neonatal group $B$ streptococcal infections in Germany. Pediatrics 117, e1139-e1145 (2006).

14. Currie, A. J. et al. Preterm infants have deficient monocyte and lymphocyte cytokine responses to group B streptococcus. Infect. Immun. 79, 1588-1596 (2011).

15. Koenig, J. M. \& Keenan, W. J. Group B streptococcus and early-onset sepsis in the era of maternal prophylaxis. Pediatr. Clin. North Am. 56, 689-708 (2009).

16. Kan, B., Razzaghian, H. R. \& Lavoie, P. M. An immunological perspective on neonatal sepsis. Trends Mol. Med. 22, 290-302 (2016).

17. Knobloch, J. et al. A systemic defect in Toll-like receptor 4 signaling increases lipopolysaccharide-induced suppression of IL-2-dependent T-cell proliferation in COPD. Am. J. Physiol. Lung Cell. Mol. Physiol. 310, L24-L39 (2016).

18. Sueyoshi, K. et al. Lipopolysaccharide suppresses $T$ cells by generating extracellular ATP that impairs their mitochondrial function via P2Y11 receptors. J. Biol. Chem. 294, 6283-6293 (2019).

19. Ufermann, C. M. et al. Indoleamine 2,3-dioxygenase activity during acute toxoplasmosis and the suppressed T cell proliferation in mice. Front. Cell Infect. Microbiol. 9, 184 (2019).

20. Saumyaa et al. Immunosuppressive property within the Streptococcus pneumoniae cell wall that inhibits generation of $\mathrm{T}$ follicular helper, germinal center, and plasma cell response to a coimmunized heterologous protein. Infect. Immun. 81, 3426-3433 (2013).

21. Goldberg, M. R. et al. Synergy between LPS and immobilized anti-human CD3epsilon mAb for activation of cord blood CD3+ T cells. Int. Immunol. 19, 99-103 (2007).

22. Carr, R. Neutrophil production and function in newborn infants. Br. J. Haematol. 110, 18-28 (2000).

23. de Jong, E., Strunk, T., Burgner, D., Lavoie, P. M. \& Currie, A. The phenotype and function of preterm infant monocytes: implications for susceptibility to infection. J. Leukoc. Biol. 102, 645-656 (2017).

24. Zhang, X., Zhivaki, D. \& Lo-Man, R. Unique aspects of the perinatal immune system. Nat. Rev. Immunol. 17, 495-507 (2017).

25. Rieber, N. et al. Neutrophilic myeloid-derived suppressor cells in cord blood modulate innate and adaptive immune responses. Clin. Exp. Immunol. 174, 45-52 (2013).
26. Elahi, S. et al. Immunosuppressive CD71+ erythroid cells compromise neonatal host defence against infection. Nature 504, 158-162 (2013).

27. Pagel, J. et al. Regulatory $T$ cell frequencies are increased in preterm infants with clinical early-onset sepsis. Clin. Exp. Immunol. 185, 219-227 (2016).

28. Pettengill, M. et al. Soluble ecto-5'-nucleotidase (5'-NT), alkaline phosphatase, and adenosine deaminase (ADA1) activities in neonatal blood favor elevated extracellular adenosine. J. Biol. Chem. 288, 27315-27326 (2013).

29. Zhu, J., Chen, H., Huang, X., Jiang, S. \& Yang, Y. Ly6C(hi) monocytes regulate T cell responses in viral hepatitis. JCl Insight 1, e89880 (2016).

30. Albertsmeier, M. et al. Monocyte-dependent suppression of T-cell function in postoperative patients and abdominal sepsis. Shock 48, 651-656 (2017).

31. Berner, R., Csorba, J. \& Brandis, M. Different cytokine expression in cord blood mononuclear cells after stimulation with neonatal sepsis or colonizing strains of Streptococcus agalactiae. Pediatr. Res. 49, 691-697 (2001).

32. Kollmann, T. R. et al. Neonatal innate TLR-mediated responses are distinct from those of adults. J. Immunol. 183, 7150-7160 (2009).

33. Levy, O. et al. The adenosine system selectively inhibits TLR-mediated TNF-alpha production in the human newborn. J. Immunol. 177, 1956-1966 (2006).

34. Giannoni, E. et al. Estradiol and progesterone strongly inhibit the innate immune response of mononuclear cells in newborns. Infect. Immun. 79 2690-2698 (2011).

35. Roger, T. et al. High expression levels of macrophage migration inhibitory factor sustain the innate immune responses of neonates. Proc. Natl Acad. Sci. USA 113 E997-E1005 (2016)

36. Heinemann, A. S. et al. In neonates S100A8/S100A9 alarmins prevent the expansion of a specific inflammatory monocyte population promoting septic shock. FASEB J. 31, 1153-1164 (2017).

37. Patera, A. C. et al. Frontline science: defects in immune function in patients with sepsis are associated with PD-1 or PD-L1 expression and can be restored by antibodies targeting PD-1 or PD-L1. J. Leukoc. Biol. 100, 1239-1254 (2016).

38. Giesbrecht, K., Former, S., Sahr, A., Heeg, K. \& Hildebrand, D. Streptococcal pyrogenic exotoxin A-stimulated monocytes mediate regulatory $\mathrm{T}$-cell accumulation through PD-L1 and kynurenine. Int. J. Mol. Sci. 20, 3933 (2019).

39. Engen, S. A. et al. The regulatory role of the oral commensal Streptococcus mitis on human monocytes. Scand. J. Immunol. 87, 80-87 (2018).

40. Gollwitzer, E. S. et al. Lung microbiota promotes tolerance to allergens in neonates via PD-L1. Nat. Med. 20, 642-647 (2014).

41. Veglia, F., Perego, M. \& Gabrilovich, D. Myeloid-derived suppressor cells coming of age. Nat. Immunol. 19, 108-119 (2018).

42. Grozdics, E. et al. B7 costimulation and intracellular indoleamine 2,3-dioxygenase expression in umbilical cord blood and adult peripheral blood. Biol. Blood Marrow Transplant. 20, 1659-1665 (2014).

43. Natarajan, G. et al. Nitric oxide and prostaglandin response to group B streptococcal infection in the lung. Ann. Clin. Lab. Sci. 37, 170-176 (2007).

44. Smith, P. L. P. et al. Peripheral myeloid cells contribute to brain injury in male neonatal mice. J. Neuroinflammation 15, 301 (2018).

45. Zaramella, P. et al. Innate immunity ascertained from blood and tracheal aspirates of preterm newborn provides new clues for assessing bronchopulmonary dysplasia. PLOS ONE 14, e0221206 (2019).

46. Malek, T. R. The biology of interleukin-2. Annu. Rev. Immunol. 26, 453-479 (2008).

47. Wood, H. et al. Breastfeeding promotes early neonatal regulatory T-cell expansion and immune tolerance of non-inherited maternal antigens. Allergy 76, 2447-2460 (2021).

48. Kostlin, N. et al. Granulocytic myeloid-derived suppressor cells from human cord blood modulate T-helper cell response towards an anti-inflammatory phenotype. Immunology 152, 89-101 (2017).

49. Gille, C. et al. Phagocytosis and postphagocytic reaction of cord blood and adult blood monocyte after infection with green fluorescent protein-labeled Escherichia coli and group B Streptococci. Cytom. B Clin. Cytom. 76, 271-284 (2009).

\section{AUTHOR CONTRIBUTIONS}

Substantial contributions to conception and design: K.M., C.G., N.K.-G. Acquisition of data or analysis and interpretation of data: K.M., H.R., J.S., S.D., B.S., L.H. Drafting the article or revising it critically for important intellectual content and final approval of the version to be published: K.M., C.F.P., N.K.-G.

\section{FUNDING}

This work was supported by a grant from the Medical Faculty of University of Tübingen (PATE, F.1275178.1 and IZKF 2019-1-12) and the German Center for 
Infection Research (DZIF) and the Ministry for Science, research and art BadenWürttemberg. Open Access funding enabled and organized by Projekt DEAL.

\section{COMPETING INTERESTS}

The authors declare no competing interests.

\section{ETHICS APPROVAL AND CONSENT TO PARTICIPATE}

Parents of newborns gave written informed consent.

\section{ADDITIONAL INFORMATION}

Supplementary information The online version contains supplementary material available at https://doi.org/10.1038/s41390-021-01880-1.

Correspondence and requests for materials should be addressed to Christian Gille.

Reprints and permission information is available at http://www.nature.com/ reprints
Publisher's note Springer Nature remains neutral with regard to jurisdictional claims in published maps and institutional affiliations. (c) (i) Open Access This article is licensed under a Creative Commons Attribution 4.0 International License, which permits use, sharing, adaptation, distribution and reproduction in any medium or format, as long as you give appropriate credit to the original author(s) and the source, provide a link to the Creative Commons license, and indicate if changes were made. The images or other third party material in this article are included in the article's Creative Commons license, unless indicated otherwise in a credit line to the material. If material is not included in the article's Creative Commons license and your intended use is not permitted by statutory regulation or exceeds the permitted use, you will need to obtain permission directly from the copyright holder. To view a copy of this license, visit http://creativecommons. org/licenses/by/4.0/.

(c) The Author(s) 2022 